\title{
Transepithelial High Fluence Crosslinking for Keratoconus - Case Report of 1-year Follow-up
}

\author{
Roberto Pinelli and Zahran Yazan Amin² \\ 1. Scientific Director, Switzerland Eye Research Institute, Lugano, Switzerland; 2. Refractive Surgeon, Yazan Zahran Eye Clinic, Amman, Jordan
}

\begin{abstract}
Purpose: To evaluate the efficacy and safety of transepithelial crosslinking for halting the progression of keratoconus. Methods: Uncorrected and corrected visual acuity, simulated keratometry, corneal topography and pachymetry data were evaluated at baseline and 1 year after bilateral transepithelial crosslinking using ParaCel ${ }^{\mathrm{TM}}$ and the $\mathrm{KXL}^{\mathrm{TM}}$ device (Avedro, Waltham, Massachusetts, US). Results: The keratometry, uncorrected visual acuity, best corrected visual acuity at the baseline and after 1 year of follow-up was stable and even improved with time. Conclusion: Transepithelial crosslinking can safely and effectively halt the progression of keratoconus as demonstrated by follow-up.
\end{abstract}

\section{Keywords}

Keratoconus, transepithelial high fluence crosslinking

\begin{abstract}
Disclosure: Roberto Pinelli and Zahran Yazan Amin have no conflicts of interest to declare. No funding was received in the publication of this article. Acknowledgements: This case report was originally published in Amin ZY and Pinelli R, Transepithelial High Fluence Crosslinking for Keratoconus, Interdiscip J Microinflammation, 2014;1:1 and has been reproduced in the European Ophthalmic Review for the ophthalmology audience with permission from the publisher and authors. Open Access: This article is published under the Creative Commons Attribution Noncommercial License, which permits any non-commercial use, distribution, adaptation and reproduction provided the original author(s) and source are given appropriate credit.

Compliance with Ethics Guidelines: Informed consent was received from the patient involved in the case study.

Received: 18 September 2014 Accepted: 10 December 2014 Citation: European Ophthalmic Review, 2015;9(1):21-2 DOI: 10.17925/EOR.2015.09.01.21

Correspondence: Roberto Pinelli, Riva Paradiso 2, Lugano 6900, Ticino, Switzerland. E: pinelli@seri-lugano.ch
\end{abstract}

Keratoconus is a non-inflammatory cone-like ectasia of the cornea, which is usually bilateral and progresses over time with consequent central and paracentral thinning of the stroma and irregular astigmatism.

The only known treatment for arresting the progression of the keratoconus is collagen corneal crosslinking, which enforces a human cornea and links the collagen with means to rejuvenate the corneal tissue. The two most widespread techniques for crosslinking are EPI-ON (transepithelial crosslinking protocol) and EPI-OFF (crosslinking with epithelium removal).

The main advantages of the transepithelial (TE) crosslinking technique over the traditional technique are that it is pain free with no stromal oedema and provides the possibility to treat both eyes at the same session. It also has the morphological advantages of reducing the rate of death of keratocytes and the number of endothelial cells. ${ }^{1}$ With the improvement of this technique it could be the standard treatment for keratoconic patients.

\section{Case Report}

A 28-year-old male patient diagnosed with keratoconus and already treated in the left eye with EPI-ON (without removing the epithelium) crosslinking in another clinic. He presented at our clinic seeking treatment in January 2013. At the time of examination his uncorrected visual acuity (UCVA) in the right eye was 20/32 best corrected visual acuity (BCVA) to 20/25 with the following refraction (cyl -0.75 axis 70 ) and left eye was 20/63 BCVA to 20/32 with the following refraction (sphere +1.25 cyl -2.5 axis 90 ). The corneal topography was performed and showed inferior steeping with cone-like configuration more in the left eye as shown in Figure 1.

The central keratometry was K1 (44.43), K2 (45.69) for the right eye and K1 (49.29), K2 (51.25) for the left eye. Central pachymetry was performed, which was 440 micron for the right eye and 469 micron for the left. Biomicroscopy showed only seborrheic blepharitis. Endothelial morphology and cell density were normal; retinal tomography was normal, tonometry was normal.

The patient was then booked for TE crosslinking, which is the usual practice in our institute and took place at the end of January 2013. We performed osmotic crosslinking using ParaCe ${ }^{\mathrm{TM}}$ riboflavin developed by Dr Roberto Pinelli, which comprises 0.25 \% riboflavin, methyl cellulose, hypotonic $\mathrm{NaCl}$ and benzalklonium chloride produced by Avedro Watham, together with the KXL device, which was also produced by the same company. The technique was as follows:

- Five minutes pre soak;

- KXI Avedro ultraviolet (UV) A source;

- ParaCel instillation every 20 seconds during UVA exposure;

- 2.40 minutes timing of exposure;

- $45 \mathrm{~mW} / \mathrm{cm}^{2}$; and

- Pulsed UVA emission.

The patient was checked immediately and at the end of the day for any eventual complications, ${ }^{1}$ which thus far we have never had. He 


\section{Figure 1: Corneal Topography}

Left Eye Pre and Post Op

A

B
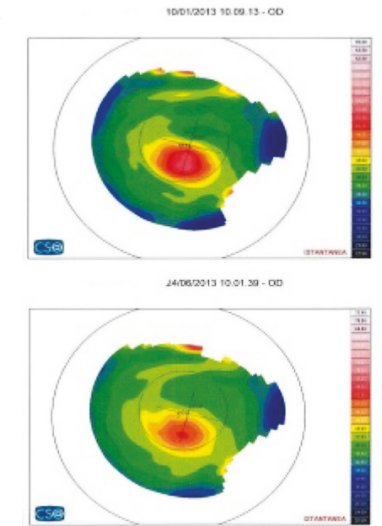

B1

A1
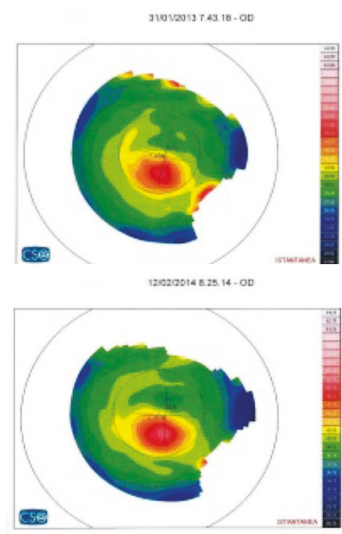

was checked again the following morning and then programmed for sequential follow-up.

The patient was checked for follow-up after 1 year because he was living so far from the clinic (Australia). During this follow-up the UCVA, BCVA of the right eye remained the same with the same refraction but the UCVA, BCVA of the left eye improved to 20/50, 25/25, respectively, with the following refraction (+1.25 cyl -1.5 axis 90). The keratometry showed changes in the both eyes as follows OD K1 (43.48) K2 (44.75) OS K1 (47.22) K2 (49.65). The pachymetry remained the same and the cornea was clear.

\section{Results}

From the results of visual acuity and keratometry of this case we can see the improvement of the visual acuity plus the decrease and the stabilisation of the keratometry of the this patient over 1-year follow-up.

\section{Discussion}

Corneal collagen crosslinking is already a well-established clinical treatment, which was originally indicated for ectatic corneal lesions such as keratoconus, pellucid marginal degeneration and ectasia post excimer laser refractive surgery. ${ }^{2}$ The main streams in the treatment are the Dresden protocol or EPI-OFF with all the pros and cons $^{2,3}$ and the EPI-ON that has gained wide development over the years. ${ }^{4-7}$ Debate continues as to whether the TE is effective and durable compared with the Dresden protocol. In our practice we use the Osmotic TE with Paracel and KCL device and the results ${ }^{6}$ have thus far been very good. There are also several other surfactant riboflavin solutions ${ }^{8}$ on the market but still quite a good number of colleagues performing this procedure who sceptical in terms of the efficacy of the protocol. There is recent attention in terms of the possible application of the technique to low power refractive errors ${ }^{8}$ as well as keratitis. The recent studies show the TE high fluence accelerated crosslinking ${ }^{9,10}$ to be safe and effective in halting the progressing of ectatic diseases. Therefore in light of these and other possible indications we feel that an effort should be made to propose a valid protocol that can lead to comparison of data in what could be another tool in refractive surgery some time later.
1. Mastropasqua L, Nubile M, Lanzini M, Morphological modification of the cornea after standard and transepithelia corneal cross-linking as imaged by anterior segment optical coherence tomography and laser scanning in vivo confocal microscopy, Cornea, 2013;32:855-61.

2. Pinelli R, Holcomb C3-R treatment opens new frontiers for keratoconus and corneal ectasia, Eyeworld, 2007;34-6.

3. Koller T, Mochen M, Seiler T, complications and failure after corneal crosslinking, I Cataract surgery, 2009,38:1358-62.

4. Filipello M, Stagni E, O'Brait D, transepithelial corneal cross

linking bilateral study, I Cataract Refract Surg, 2012:38:283-91.
5 Wollensack G, Spoerl E, Seiler T, Riboflavin/ultraviolet-A and collagen induced collagen crosslinking for the treatment of keratoconus, Am J Ophthalmol, 2003;135:620-7.

6 Caporossi A, Baioccchi S, Mazzotta C, et al., Parasurgical therap for keratoconus by riboflavin-ultraviolet type A rays induced crosslinking of corneal collagen: Preliminary refractive results in an Italian study, J Cataract Refract Surg, 2006;32:837-45.

7. Kopen C, Wonters K, Mothysen D, et al., Refractive and topografic results of benzalkonium chloride-assisted transepithelial crosslinking, I Cataract Refract surg, 2012;38:1000-5.
8. Kanellopoulos AJ, Long term results of a prospective randomised bilateral eye comparison trial of higher fluence shorter duration ultraviolat A radiation and riboflavin crosslinking for progressive keratoconus, Clin Ophthalmol, 2012;6:97-101.

9. Tomita M, Mita M, Huseynova T, Accelerated versus conventional corneal collagen crosslinking, I Cataract Refract Surg, 2014;40:1013-102.

10. Hersh P, Lesniak $S$, Transepithelial corneal collagen crosslinking for keratoconus: Six-month results, I Cataract Refract Surg, 2014;40:1971-9 